\title{
CDLN - CLUSTER DISTANCE BASED DATA FORWARDING AND OPTIMAL LEADER ELECTION USING FUZZY INFERENCE IN WIRELESS NETWORK
}

\author{
Sachidanand S Joshi \\ Assistant Professor, Department of Information Science \&Engineering \\ SDM College of Engineering \& Technology \\ Dharwad, Karnataka, India \\ Email: sachinjoshi055@gmail.com \\ Sangappa Ramachandra Biradar \\ Professor, Department of Information Science \&Engineering \\ SDM College of Engineering \& Technology \\ Dharwad Karnataka,India \\ Email: srbiradar@gmail.com
}

\begin{abstract}
:
Wireless networks in general can consume a lot of energy and incur losses if they do not have the right protocols to properly add packets to the destination. Similarly, if the connectivity of the network nodes is different, its resource losses will be higher. Thus, the network will quickly disconnect and cause very high losses. As well as wireless nodes, this sends various data, so energy losses are high. This proposed method CDLN - cluster distance-based data forwarding and optimal leader election using fuzzy inference in wireless network -is designed to take care of all these and formulate routing accordingly. In this method, the cluster set is formed and the main leader and assistant leaders are selected. Thus, when the main leader is disconnected, the assistant cluster acts to prevent network loss. Because the transmission ranges in this network are different sizes, the cluster set will have different members. These cluster sets are formed in a fuzzy inference mode and set up with precise leaders and routing, thus reducing energy costs, network life time and the number of dead nodes. It can also be seen in the output that the network lasts longer.
\end{abstract}

Keywords: Main Leader; Assistant Leader; Various Data; Energy reduction; Cluster Routing.

\section{Introduction}

Wireless Adhoc communication is now widely used in most products, city and remote monitoring, as well as patient monitoring in the medical field. Wireless communications are used in many places today. Adhoc networks is a method of creating locations where sudden wireless connections are required. This requires wireless devices such as laptops and tablets. Without any infrastructure connection, the nodes will communicate within them. Thus, these require proper protocols to communicate. Those protocols should focus on neighbor selection and routing to make connections. Those protocols explore the metrics of the network and enable the corresponding routing properties to help the network run smoothly.

In this proposed network, we make neighbor selection, cluster set formation, leader election, taking into account certain factors when making connections between nodes. In the cluster set thus formed, the leader selection and the transmission range of that cluster set are accurately calculated and then selected so that the correct leader and transmission range can be found. Also, we select two leaders in the selected cluster set. Thus, by the time the main leader in the network completes its operation, without losing the connection in the network, the assistant leader will start to be created immediately, at that time the selection of the new main leader will start at the end of the next cluster duration. This will reduce network connection delays and reduce data loss.

The fuzzy inference system is used to form the cluster set. All the selected parameters are given as inputs for fuzzification. Then all the given inputs are converted to fuzzy value. Thereby, those fuzzy values are given a required truth value. Then, we form the fuzzy rule set, find the fluctuations in those values, select the best output and select it as the transmission range and leader, this saves network resources. 
In this paper, in section 2; we look at previous research on this network system as a literature survey. In section 3 , we see the proposed method, CDLN -cluster distance-based data forwarding and optimal leader election using fuzzy inference in wireless network, in detail. In section 4, the results and discussion are described. Finally, section 5 concludes on this protocol.

\section{Literature Survey}

Unfortunately, random mobility represents more of a problem to solve than an advantage to exploit. A network access point mounted on a means of public transportation that moves with a periodic schedule represents a case of predictable mobility [3]. We refer the stream of data to as data flow and the node originating it as the source. To control the energy, all nodes readings are combined and then processed through the point of aggregation. This forwarded single message contains the reading got from various nodes which is then aggregated value [1]. The routing part includes the sender broadcasting $\mathrm{H}$ times to guarantee an assured delivery rate, each receiver calculating its probability to forward the message to reduce the number of replicated messages, and the updating of packet loss rates of links [10]. The data generated by nodes in the wireless network is too much for the enduser to process so they require methods to combine them into a small set of meaningful information. A simple way is data aggregation from different nods and a more elegant approach is data fusion which can be defined as a combination of several unreliable data measurements to produce a more accurate signal by enhancing the common signal \& reducing uncorrelated noise [14]. The remarkably high variance among the residual energies is because of the different distances of each node from the sink and, in general, to the different number of node-todestination routes to which a node belongs, which implies a different number of packets to relay. Nodes along the "cross" centered at the sink tend to be the preferred data relays [2]. Finding the best route only with the shortest distance consideration may lead to network partitioning. Finding the best route only based on energy balancing consideration may lead to a long path with high delay and decreases network lifetime [16]. The Random ReRouting algorithm which is the focus of this paper offers a real-time adaptive capability to detect unusual events and provide them with significantly better QoS in the presence of larger volumes of routine data traffic. We have provided both analyses based on diffusion approximations and simulation to show its strong potential to satisfy the specific needs for differentiated QoS in WSNs [21]. Packet forwarding is a typical means for nodes to proficiently impart their packets to one another. The packet forwarding mechanism can be used to introduce the system plan for route discovery between a given pair of nodes and could serve as a productive strategy to confine nodes [18]. While the nodes have limited sensing region, processing power, and energy, networking a large number of such nodes gives rise to a robust, reliable, and accurate wireless network covering a wider region. Since the nodes are energy-constrained, a typical deployment of a WSN poses many challenges and causes energyawareness at all layers of the networking protocol stack [4]. This provides an optimal path between source and destination. Selecting an optimal path between the source and destination and sending the data through that path may not increase the lifetime of the network. The energy usage in such an approach is not as efficient as that in the multi-path routing approaches [19].

Hierarchical routing also known as cluster-based routing subdivides the network into small groups and routing is performed hierarchically. In location-based routing protocols, the nodes are addressed according to their location [9]. The largest magnitude Eigen value of the probabilistic connectivity matrix was proved to be a good measurement of network connectivity. However, the major focus of the literature works mentioned above is on the connectivity between any two nodes within the network [5]. The nodes in the network send their data to the base station from where the end user can access the sensed data. As most of the battery power is utilized in data transmission between nodes and base station the routing algorithms must be designed to increase lifespan of nodes in the network in order to improve the overall network performance [13]. To establish the routing process, the proposed protocol takes advantage of a distributed intelligent technique inspired by the collective behaviors of ants in nature. It establishes energy-efficient paths, taking into consideration, at each round, the energy state of the whole network [6]. This localization scheme can be simply implemented in ad-hoc networks without adding extra nodes or equipment's. However, if the nodes are sparsely distributed in the networks, the location estimation may be very coarse because the hop count is inaccurate [20]. The PSOR does routing by taking energy as a fitness value. By calculating the fitness value of the nodes, the protocol finds a new path to route the packets. From the various paths found it selects the optimized one that consumes less energy to route the packets [8]. Location-based routing usually uses a greedy forwarding mechanism to forward a data packet from source to destination. Greedy approach forwards packets to the neighbor, which is closest to the destination. It assumes that the network is sufficiently dense; each node has its own accurate location information, its neighbors' locations, and high link reliability [15]. The performance of CARP has been compared to that of FBR and of an enhanced version of flooding, called EFlood, where nodes wait for a random time before forwarding the packet. We consider networks with desirable size, varying traffic and packet size [7]. Interestingly, in many MWSN applications, the mobile elements are naturally available in the sensing field. For instance, animals in habitat monitoring or soldiers in battlefield observing applications can carry the nodes and play as the mobile nodes; moreover, vehicles can be exploited as the mobile sinks in a WSN to observe traffic conditions [11]. Transmission of imaging data requires 
careful handling in order to ensure that end-to-end delay is within acceptable range. Such performance metrics are usually referred to as quality of service (QoS) of the communication network [17]. The source of energy in node is a main constrain among them. It can be minimized by increasing the density of energy in conventional energy sources. One of the elegant ways to prolong the lifetime of the network is to be reduced energy consumption in nodes [12].

\section{CDLN - Design and Implementation}

\subsection{Network Initiation Model}

Once the placement of the nodes in the mobile Adhoc network is over, each node will begin to broadcast a node initiation message to start the communication. Information about that node will be attached to the message. These are node ID, locationL, energyE, etc. Also, the hop $\operatorname{countH}_{\mathrm{C}}$ of this node is indicated at 1 . The neighboring node that receives this broadcast message will update the information it received, then increase its $_{\mathrm{C}}$ and reply back to that node. Thus, across the network, all nodes exchange information and make an initial introduction. Such a wayD number of nodesd $d_{1} \ldots d_{n}$ placed on the network region and each node has $N=n_{1} \ldots n_{n}$ number of neighbours. Here, Ddefines the devices on the network. Similarly, all nodes will mark its locationL, $\mathrm{E}, \mathrm{H}_{\mathrm{C}}$ and send an introductory link message across the transmission region $\mathrm{T}_{\mathrm{R}}$. Accordingly, each node will receive the message and update the neighbor's metrics. Also, note the $\mathrm{H}_{\mathrm{C}}$ for that node and compute distance $\mathrm{D}_{\text {ist }}$ from node to $n_{i}$ as $D_{S \rightarrow n}$. Thus, the entire network is connected. Defines the network region $N_{R}$ considered from $T \times T$.

$\mathrm{D}_{\mathrm{S} \rightarrow \mathrm{n}_{\mathrm{i}}}=\int \mathrm{N}_{\mathrm{R}} \sqrt{(\mathrm{X}+\mathrm{Y})^{2}} \frac{\mathrm{T}}{\mathrm{N}_{\mathrm{R}}} \Delta \mathrm{N}_{\mathrm{R}}$

$$
\mathrm{P}_{\mathrm{N}}=\sum \mathrm{D}_{\mathrm{S} \rightarrow \mathrm{n}_{\mathrm{i}}}
$$

The network contains mobile nodes

The node is placed in the topography of the network regionN $N_{R}=\mathrm{T} \times \mathrm{T}$

Nodes are randomly dispersed in a two-dimensional network regionX, andY.

All nodes are connected with battery for energy management

At beginning all nodes contains the equal energy

The nodes transmission power adjusted as per the $T_{R}$

Cluster leader use the data union process to minimize the energy usage and resources

The transmission range $T_{R}$ of the node is $\frac{T}{\sqrt{\mathrm{ID}}}$. The number of nodes within the neighbor coverage of the network varies from time to time. The number of such changing neighbors $\mathrm{C}_{\mathrm{N}}$ is calculated as follows within the circular $\mathrm{T}_{\mathrm{R}}$.

$$
\mathrm{C}_{\mathrm{N}}=\frac{2 \mathrm{~T}}{\sqrt{\pi \mathrm{D}}}
$$

The neighboring coverage diameter and the circular radius of the network are known by $\mathrm{C}_{\mathrm{N}}$.

\subsection{Network Resource Validations}

The energy consumption of nodes running in a network has two parts. They are, transmission power $\mathrm{T}_{\mathrm{P}}$ and receiving powerR $R_{P}$. This energy helps the electrical usage of the node and its spare parts to function along with channel operations.

$$
\begin{gathered}
\mathrm{R}_{\mathrm{P}}=\mathrm{E}_{\text {lec }} \mathrm{P}_{\mathrm{S}} \mathrm{P}_{\mathrm{C}} \\
\mathrm{T}_{\mathrm{P}}=\left(\left(\mathrm{E}_{\text {lec }}+\mathrm{E}_{\text {CU }} \mathrm{D}_{\text {ist }}+\mathrm{E}_{\mathrm{V}}\right) \mathrm{P}_{\mathrm{S}} \mathrm{P}_{\mathrm{C}}\right)
\end{gathered}
$$

Here, $E_{V}$ is the energy variations occurrence according the channel state. According to the transmission and receiving packets among nodes, energy consumption $E_{\mathrm{V}}$ computed as below:

$\mathrm{E}_{\mathrm{V}}=\mathrm{SE}_{\text {lec }}+\mathrm{SE}_{\mathrm{UC}}+\mathrm{R}_{\mathrm{P}}+\mathrm{E}_{\mathrm{U}}+\mathrm{D}_{\mathrm{S} \rightarrow \mathrm{B}+} \mathrm{P}_{\mathrm{S}} \mathrm{P}_{\mathrm{C}}$

Here, $P_{S}$ and $P_{C}$ are the packet size and of packet counts of data transmission in order, also $E_{\text {lec }}$ and $E_{C U}$ are the electrical usage for the components and electrical usage for channel. Similarly, $\mathrm{D}_{\text {ist }}$ is the distance between the nodes are computed as below: $\mathrm{TheD}_{\text {ist }}$ is calculated according to the Pythagorean Theorem, here, location of the node iis $\left(\mathrm{X}_{1}, \mathrm{Y}_{1}\right)$, and neighbor location $\mathrm{j}$ is $\left(\mathrm{X} 2, \mathrm{Y}_{2}\right)$,

$$
D_{\text {ist }}=\sqrt{\left|X_{1}-X_{2 \mid}^{2}+\right| Y_{1}-Y_{2 \mid}^{2}}
$$


Here, the size of the hello message used to find the neighbor, the messages used to select the cluster, and the messages used to select the path and the size of the data packets vary. Therefore, their travel time will also vary.

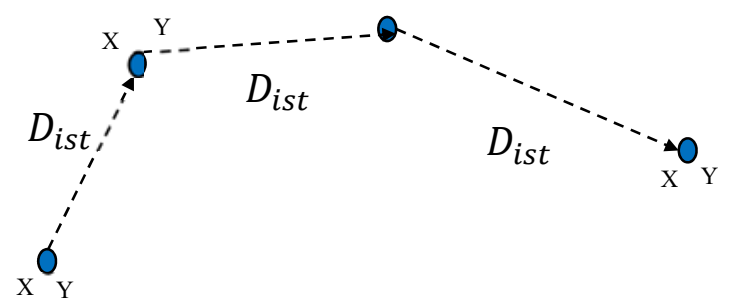

All these should be taken into account during energy calculation. During data transmission, here the nodes form the path in cluster mode. Therefore, in each cluster, a cluster leader and cluster members are selected. The selected cluster sets run in parallel. If more than one node sends the same data at the same time, only one piece of data will be combinedD $\mathrm{D}_{\mathrm{C}}$ with the ID of those nodes and $\mathrm{CH}$ will be sent to the next $\mathrm{CH}$. This will also save network resources, resources include energy, bandwidth, and channel utilization.

$$
\mathrm{D}_{\mathrm{C}}=\frac{\mathrm{D}_{\mathrm{CC}}}{\mathrm{B}_{\mathrm{C}}}
$$

Here, $D_{C}$ is the combined data during transmission, and $D_{C C}$ is the data combined counts, also, $B_{C}$ is the total transmission bit counts transferred during communication. Here, $\mathrm{E}_{\mathrm{U}}$ is the energy usage during data combined transmission in the network.

$$
\mathrm{E}_{\mathrm{U}}=\mathrm{D}_{\mathrm{C}} \mathrm{P}_{\mathrm{S}} \mathrm{P}_{\mathrm{C}}
$$

$\mathrm{E}_{\mathrm{U}}$ Calculation will also save network resources. Resources include energy, bandwidth, and channel utilization.

\subsection{Cluster Communication in Network}

From the nodes in the neighbor set, select one node as the leader of that cluster set, as well as calculate the optimal solution $\mathrm{O}_{\mathrm{S}}$ of the cluster members to be in a cluster set.

$$
\mathrm{O}_{\mathrm{V}}=\frac{\mathrm{T}}{\mathrm{D}_{\mathrm{ist} \rightarrow \mathrm{P}_{\mathrm{N}}}} \frac{\mathrm{E}_{\mathrm{CU}}}{\mathrm{E}_{\mathrm{V}}} \frac{\mathrm{N}_{\mathrm{R}}}{2 \pi}
$$

All the nodes in the network start to compete at the beginning to form the cluster set. The clusters formed here are arranged heterogeneously on different $T_{R}$ trunks according to their locations. Cluster sets near destination have the lowest $\mathrm{T}_{\mathrm{R}}$. Similarly, depending on the network size and the density of the existing nodes, their topography area will expand. Depending on the topography area, cluster sets are formed.

Depending on the distances to destination, the $T_{R}$ of that set will vary. Thus, the number of cluster members also varies depending on the $T_{R}$. This creates dissimilar cluster sets in the network. Thus, the heterogeneous cluster sets in the network and its $T_{R}$ are formed accordingly. We must select a leader from those sets. Initially all the nodes in the network will have the same volume of resources. Therefore, the initial phase cluster must be formed at random manner. Then its resource utilization will vary depending on their neighbor counts and distances between them. The initial random leader $\mathrm{R}_{\mathrm{L}}$ selection is calculated as follows.

$$
\mathrm{R}_{\mathrm{L}}=\frac{\mathrm{P}_{\mathrm{r}}}{1-\mathrm{P}_{\mathrm{r}} \mathrm{C}_{\mathrm{D}}} \frac{1}{\mathrm{P}_{\mathrm{r}}}
$$

Here, is the random probability starts from 0 to 1 . Also, is the cluster duration period, this defines new cluster formation after a period. This reformation of cluster at every particular period balances the network resources. During the second stage cluster selection, the leader election is done in fuzzy optimization mode.

\subsection{Leader Election in Network}

Three parameters are given as inputs of the fuzzification process during leader selection. These are the $\mathrm{N}_{\mathrm{D}}, \mathrm{E}_{\mathrm{C}}$, and the distance from source to destination $\mathrm{D}_{\text {ist } \rightarrow \mathrm{BS}}$.

$$
\mathrm{N}_{\mathrm{D}}=\mathrm{N}_{\mathrm{C}}, \forall \mathrm{n}++
$$

With this function, the exact probability value for the leader and the distance to the transmission range corresponding to where it is located are obtained. These values are expressed as worst, weak, regular, less than regular, more than regular robust, very robust. When the network size and its density vary, these membership functions will reveal the exact output of the nodes accordingly. When the network size and its density vary, these 
membership functions will reveal the exact output of the nodes accordingly. We defuzzify these outputs and select the exact leader as follows as $\mathrm{D}_{\mathrm{F}}$ data forwarders.

$$
\mathrm{D}_{\mathrm{F}}=\frac{\mathrm{I}_{1}}{1-\mathrm{P}_{\mathrm{r}} \mathrm{C}_{\mathrm{D}}} \frac{1}{\mathrm{P}_{\mathrm{r}}}
$$

This leader selection will be complete by all nodes. Then calculate the time it takes to receive the parameters $\mathrm{P}_{\mathrm{PT}}$ as parameter processing time with tuning measurem, $\mathrm{n}$ of all transmission range nodes and process them in fuzzification mode as follows.

$$
\mathrm{P}_{\mathrm{PT}}=\mathrm{S}_{\mathrm{T}} \frac{\mathrm{M}_{\mathrm{AE}}}{\mathrm{R}_{\mathrm{E}}} \mathrm{m}+\frac{\mathrm{M}_{\text {CDist }}}{\mathrm{T}_{\mathrm{R}}}
$$

Here, $S_{T}$ is the stable position time of node, $R_{A E}$ is the mean remaining energy of the node within the $T_{R}$, also is the node mean cluster distance, similarly $\mathrm{m}$ and $\mathrm{n}$ are equal to 1 . A leader is selected at the end of eachP $\mathrm{PT}_{\mathrm{PT}}$ variation, as well as at the end of each cluster interval. Also, in a cluster set, two leaders are chosen as the main leader and the assistant leader. When the main leader loses the quality of its leader position, the assistant leader begins to act before the next leader election. This can reduce data loss on the network. This is because the second leader is already connected to that cluster set, so there is no chance of connection loss. By default, once a node is selected as a leader, it will start broadcasting the leader announcement message on that cluster set. Other nodes that receive this message will update the leader ID and send a join message. Perhaps, if a node happens to hear the announcement of two leaders, it will send a message to connect with the leader at the shortest distance after calculating the $\mathrm{M}_{\mathrm{CD} \text { ist }}$ between them. Thus, the qualifications of the elected leaders are considered by the rule set of fuzzy inference.

$$
\begin{aligned}
& \text { If } \mathrm{R}_{\mathrm{E}} \rightarrow \text { Mean, } \mathrm{D}_{\text {ist } \rightarrow \mathrm{P}_{\mathrm{N}}} \rightarrow \text { utmost, } \mathrm{N}_{\mathrm{D}} \rightarrow \text { MeanThen, the } \mathrm{T}_{\mathrm{R}} \rightarrow \text { Huge. } \\
& \text { If } \mathrm{R}_{\mathrm{E}} \rightarrow \text { Mean, } \mathrm{D}_{\text {ist } \rightarrow \mathrm{P}_{\mathrm{N}}} \rightarrow \text { utmost, } \mathrm{N}_{\mathrm{D}} \rightarrow \text { More Then, the } \mathrm{T}_{\mathrm{R}} \rightarrow \text { Huge. } \\
& \text { If } \mathrm{R}_{\mathrm{E}} \rightarrow \text { More, } \mathrm{D}_{\text {ist } \rightarrow \mathrm{P}_{\mathrm{N}}} \rightarrow \text { utmost, } \mathrm{N}_{\mathrm{D}} \rightarrow \text { Mean Then, the } \mathrm{T}_{\mathrm{R}} \rightarrow \text { Huge. } \\
& \text { If } \mathrm{R}_{\mathrm{E}} \rightarrow \text { More, } \mathrm{D}_{\text {ist } \rightarrow \mathrm{P}_{\mathrm{N}}} \rightarrow \text { Mean, } \mathrm{N}_{\mathrm{D}} \rightarrow \text { More Then, the } \mathrm{T}_{\mathrm{R}} \rightarrow \text { Huge. }
\end{aligned}
$$

Consider each computation is (I: $r, s, t, u)$ is

$$
\operatorname{big}\left(\operatorname{small}\left(\frac{\mathrm{I}-\mathrm{r}}{\mathrm{s}-\mathrm{r}} \cdot \frac{\mathrm{u}-\mathrm{I}}{\mathrm{u}-\mathrm{t}}\right)\right)
$$

Update big value as output and compute all metrics in this way to get the sharp highest range output value. So, finalize the huge $T_{R}$ from the computed values as follows: the specified values obtained from the above computation to know each cluster set coverage area.

Small $(0.90,0.81,0.89) \rightarrow 0.81$
Small $(0.99,0.84,0.11) \rightarrow 0.11$
Small $(0.97,0.21,0.88) \rightarrow 0.21$
Small $(0.96,0.27 .0 .17) \rightarrow 0.17$

Of all the values given above, the highest value is 0.86 . This is considered to be the maximum $T_{R}$ of the cluster set. Thus, in all cluster sets in the network, its different transmission ranges are calculated. This decrease as it is calculated closer to the $\mathrm{B}_{S}$, and increases as the distance between the node and the destination increases, or varies greatly depending on the network configuration.

\begin{tabular}{|c|c|c|c|c|}
\hline Distance & Energy & Node Degree & Transmission Range & Fuzzified output \\
\hline Near & weak & More & Very small & Very weak \\
\hline Near & weak & medium & small & weak \\
\hline Near & weak & small & Rather small & small average \\
\hline Near & mean & More & small & mean \\
\hline Near & mean & medium & Rather small & small average \\
\hline Near & mean & small & Medium small & weak \\
\hline Near & More & More & Rather small & Very robust \\
\hline
\end{tabular}




\begin{tabular}{|c|c|c|c|c|} 
Near & More & medium & small & strong \\
\hline Near & More & small & Medium small & Above mean \\
\hline Long & weak & More & Medium small & mean \\
\hline Long & weak & medium & Rather small & small average \\
\hline Long & weak & small & small & weak \\
\hline Long & mean & More & Average big & small average \\
\hline Long & mean & medium & Medium & strong \\
\hline Long & mean & small & Medium small & Above mean \\
\hline Long & More & More & Average big & mean \\
\hline Long & More & medium & Medium & weak \\
\hline Long & More & small & Medium small & Very weak \\
\hline Utmost & weak & More & big & small average \\
\hline Utmost & weak & medium & Average big & mean \\
\hline Utmost & weak & small & Medium & small average \\
\hline Utmost & mean & More & Less big & mean \\
\hline Utmost & mean & medium & big & small average \\
\hline Utmost & mean & small & Average big & Above average \\
\hline Utmost & More & More & big & Very robust \\
\hline Utmost & More & medium & Less big & Robust \\
\hline Utmost & More & small & Huge & Above average \\
\hline
\end{tabular}

At the end of each leader act duration, a leader re-election takes place. At the beginning of the network, random cluster selection takes place and the acting leader is selected. Then their different parameters are calculated and the transmission range for those clusters is calculated accurately as its diameter. The leader will then be selected. Thus, the number of leaders is reduced as the cluster is formed in different ranges. Thus, reducing the number of forwarding $\mathrm{H}_{\mathrm{C}}$. Thus, saving network resources and it is used to extend the life of the network.

Thereafter, the selection for the optimized leader will take place as follows.

IfR $_{\mathrm{E}} \rightarrow$ Mean, $\mathrm{D}_{\mathrm{ist} \rightarrow \mathrm{P}_{\mathrm{N}}} \rightarrow$ utmost, $\mathrm{N}_{\mathrm{D}} \rightarrow$ Regular Then, the $\mathrm{L}_{\mathrm{D}} \rightarrow$ regular.

If $\mathrm{R}_{\mathrm{E}} \rightarrow$ More, $\mathrm{D}_{\mathrm{ist} \rightarrow \mathrm{P}_{\mathrm{N}}} \rightarrow$ utmost, $\mathrm{N}_{\mathrm{D}} \rightarrow$ Mean Then, the $\mathrm{T}_{\mathrm{R}} \rightarrow$ Robust.

If $\mathrm{R}_{\mathrm{E}} \rightarrow$ More, $\mathrm{D}_{\mathrm{ist} \rightarrow \mathrm{P}_{\mathrm{N}}} \rightarrow$ Mean, $\mathrm{N}_{\mathrm{D}} \rightarrow$ More Then, the $\mathrm{T}_{\mathrm{R}} \rightarrow$ Robust

If $\mathrm{R}_{\mathrm{E}} \rightarrow$ More, $\mathrm{D}_{\mathrm{ist} \rightarrow \mathrm{P}_{\mathrm{N}}} \rightarrow$ Weak, $\mathrm{N}_{\mathrm{D}} \rightarrow$ More Then, the $\mathrm{T}_{\mathrm{R}} \rightarrow$ More Robust.

Then we need to find its big and small values as already discussed. Then finalize the final leader selection at second cluster duration as follows:

Small $(0.81,0.33,0.39) \rightarrow 0.33$

Small $(0.79,0.37,0.49) \rightarrow 0.37$

Small $(0.82,0.27,0.22) \rightarrow 0.22$

Small $(0.870 .28 \cdot 0.27) \rightarrow 0.27$

Here, of all the given values, 0.37 is the maximum value. Due to this, the leader selection will be the node that sticks to this number. Thus, only the node that gives accurate outputs on all the selected parameters will run as the leader. Also, the node in the second place will run as the assistant leader. 


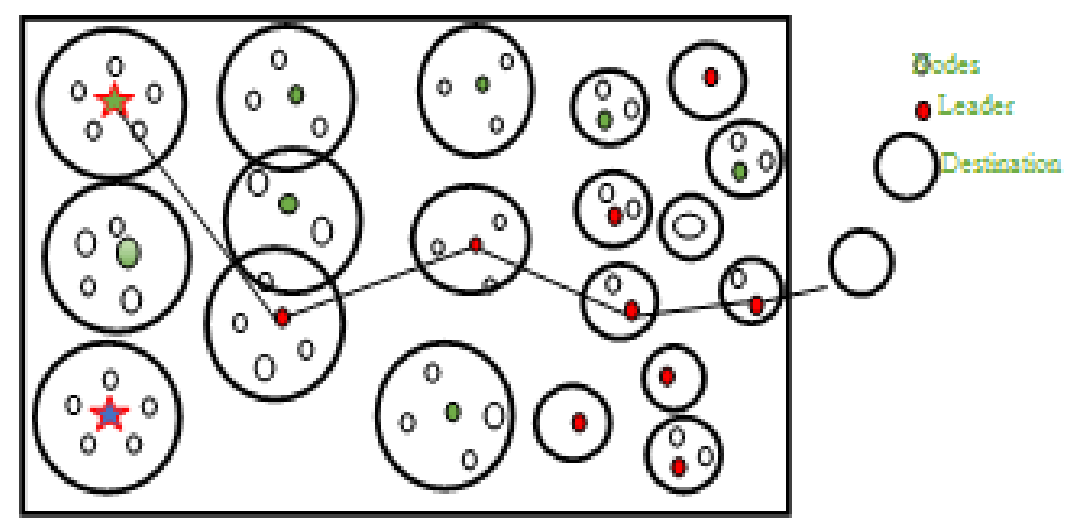

Figure. 1 Cluster Routing

\subsection{Routing Through Ladders}

Once the cluster formed with accurate leader as shown in Figure.1, after this election, during routing, we deliver data from one leader to another leader by contacting the destination then, in the path selection, the distance to one leader and another leader and its angle are calculated and delivered to the data destination by the leader at the shortest distance. For this, the angle and direction between the leaders are calculated and the path is set through it. Once two cluster sets are selected, the minimum probability and maximum probability for their interactions are calculated and its distances are determined.

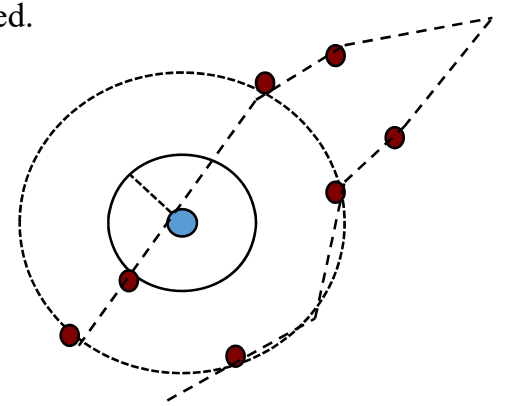

Figure.2 Transmission Ranges

The short-dotted line of the leader indicates its transmission distance and its longest dotted line indicates its communication distance as shown in Figure.2. A leader can summarize the distance of this circular direction and the distance of the destination to communicate with another leader and thereby find their total distance $T_{D}$ and their circumference. We select the shortest route and finally deliver the data. Their total diameter $\mathrm{D}_{\mathrm{M}}$ is calculated as follows. Since we have formed cluster sets of different sizes, this circumference will be optimal.

$$
\begin{gathered}
\sum \mathrm{T}_{\mathrm{D}}=\mathrm{D}_{\text {ist } 1}+\cdots \mathrm{D}_{\text {ist2 }}+\mathrm{D}_{\text {istn }} \\
\mathrm{D}_{\mathrm{M}}=\pi \mathrm{T}_{\mathrm{R} 1}{ }^{2}+\cdots \pi \mathrm{T}_{\mathrm{Rn}}{ }^{2}
\end{gathered}
$$

\section{Results and Discussions}

Here, the network is selected in the size of $500 \mathrm{x} 500$ square meters in which 100nodes are randomly placed with mobility. In it, the constant bit rate application is connected and the nodes started see it to the destination. The path selection required for this is described here. Table 1 contains the used parameters of the network.

\begin{tabular}{|l||l|}
\hline Parameter & Value \\
\hline \hline Traffic Type & CBR-Constant Bit Rate \\
\hline \hline Nodes & 100 \\
\hline \hline Propagation Model & Two Ray Ground \\
\hline \hline Antenna & Omni-Directional \\
\hline \hline Network Size & $500 \times 500$ \\
\hline
\end{tabular}

Table.1 Network Parameters 


\section{Remaining Energy}

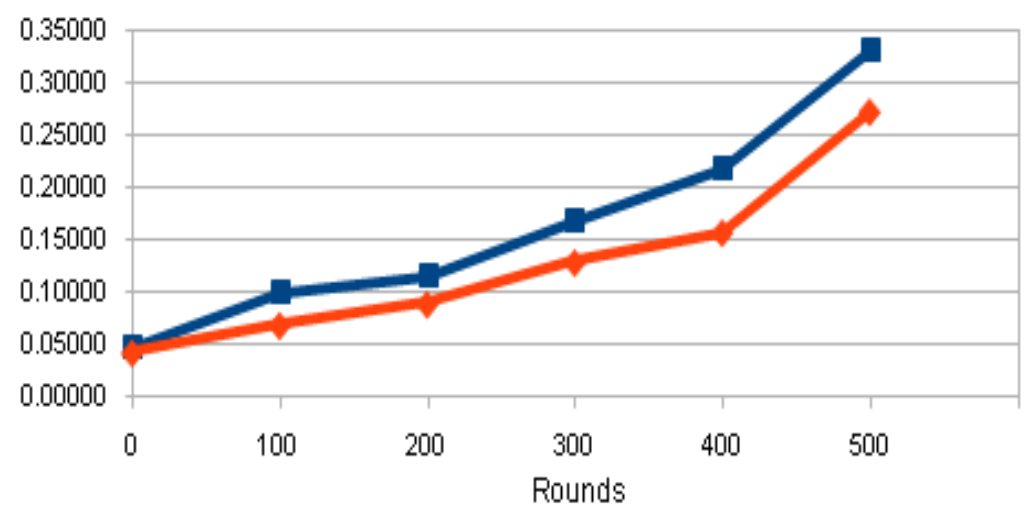

Figure.3 Rounds vs. Remaining Energy

\section{Dead rounds}

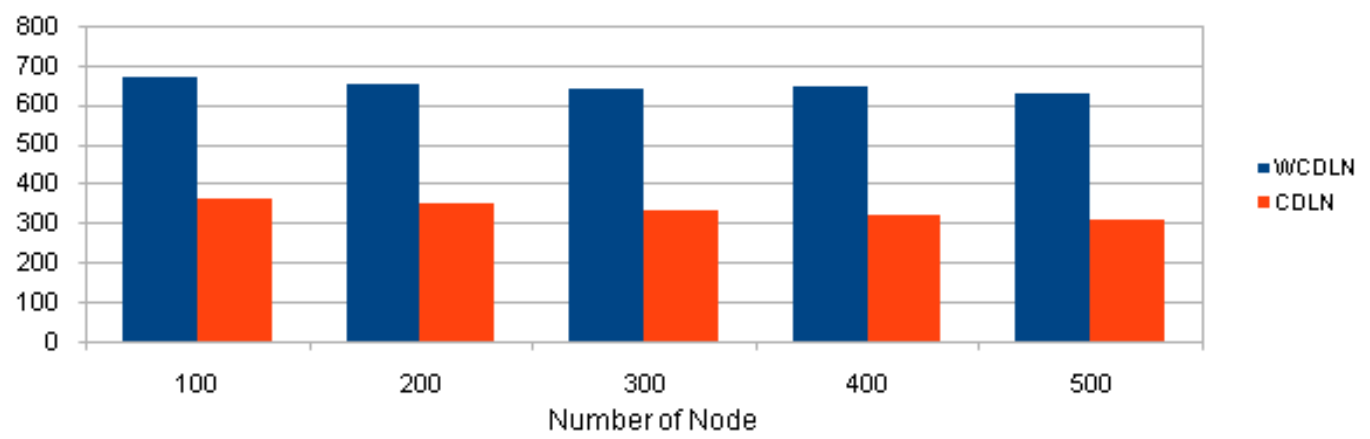

Figure.4 Node vs. Dead Rounds

Alive Nodes

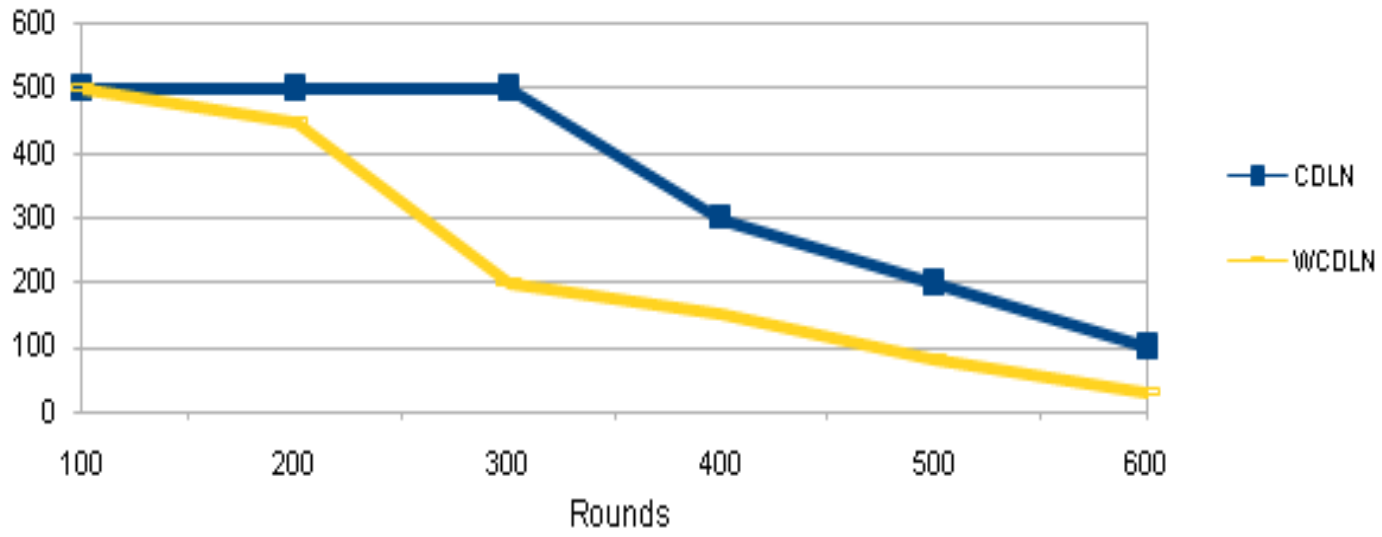

Figure.5 Rounds vs. Alive Nodes 


\section{Remaining Energy $(J)$}

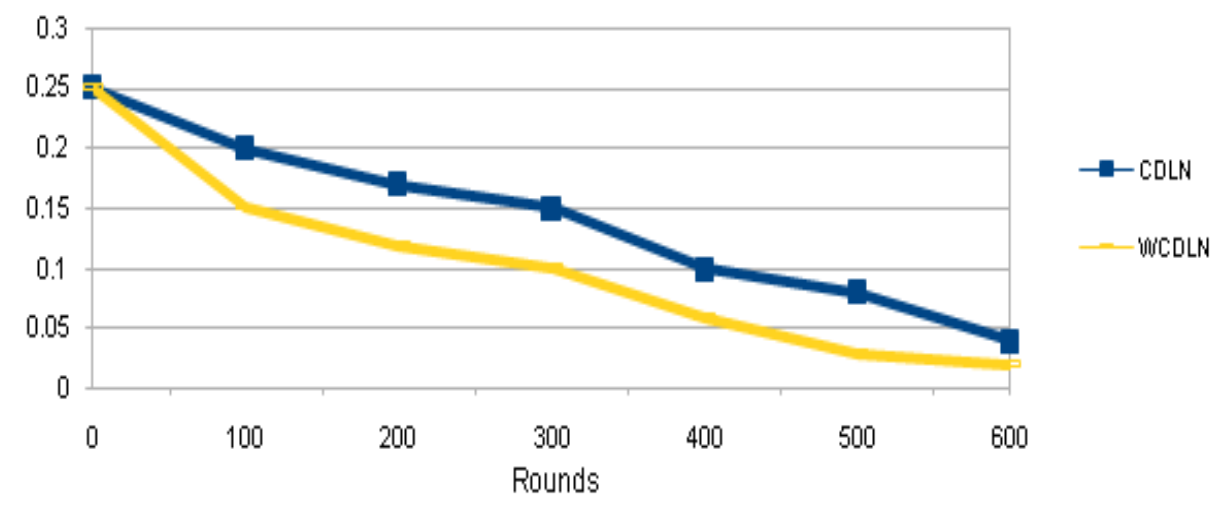

Figure.6. Average Remaining Energy

In these graphs, their losses, remaining energy, and nodes are calculated according to the energy of the nodes. Thus, we can see that the resources of the network have been turned off and the system has been used. From this we realize that the distance of the nodes, their transmission range and cluster members count are at the right level. If the number of dead nodes in the network is low and the number of living nodes is high, the protocol running on that network can be assumed to run with accurate calculations. Due to this we see that the remaining energy of the network has increased. From Figure.3 to Figure 6, all the results are tested according to the changes in the cluster forming rounds and the number of nodes. Thus, we can see more living nodes, as well as more remaining energy and less dead nodes. This is an expression of the quality of measures.

\section{Conclusion and Future Work}

In this network, the nodes are randomly placed and integrated by wireless propagation. All those nodes are formed by their optimal transmission range, cluster set, by calculating energy, neighbor count, distance between them and their transmission range. Similarly, in fuzzy optimization mode, the leader election takes place. Thereafter, in the leader-to-leader communication mode, routing is done. In that routing, the distance and circumference of the communication between the leaders are taken into account and the data is sent along the path where they have the lowest values. Thus. It can be seen that their energy level is very low.

In the future, same network we can focus on the Mac layer along with cognitive channel optimization, we can further reduce network delay and save network resources and prolong the life of the network.

\section{References}

[1] Pritesh A.Patil, R.S. Deshpande, "Trustworthy Routing in Wireless Networks Using Hop Count Filter", International Journal of Innovative Technology and Exploring Engineering (IJITEE), Volume-8 Issue-5 March, 2019.

[2] Stefano Basagni, Alessio Carosi and Chiara Petrioli, "Mobility in Wireless Networks".

[3] Enrico Natalizio and Valeria Loscr, "Controlled Mobility in Mobile Networks: Advantages, Issues and Challenges",19 Dec 2013.

[4] Jaydip Sen, "An Adaptive and Multi-Service Routing Protocol for Wireless Networks".

[5] Liao Wenxing, Wu Muqing, Zhao Min, Li Peizhe and Li Tianze, "Hop count limitation analysis in wireless multi-hop networks",International Journal of Distributed Networks, 2017.

[6] Hana Rhim, Karim Tamine, RymaAbassi, Damien Sauveron andSihemGuemara, "A multi-hop graph-based approach for an energyefficient routing protocol in wireless networks",Human-Centric Computing and Information Sciences,2018.

[7] Stefano Basagni, Chiara Petrioli, Roberto Petroccia, Daniele Spaccini, "CARP: A Channel-aware routing protocol for underwater acoustic wireless networks"Ad Hoc Networks, 2015.

[8] A. Balamurugan, "An Energy Efficient Fitness based Routing Protocol in Wireless Networks".

[9] Abhinav Valada, David Kohanbash, George Kantor, "DSRP: Distributed Web Routing Protocol".

[10] Xiao Chen, Zanxun Dai, Wenzhong Li, Hongchi Shi, “A Layer-Based Routing Protocol for Heterogeneous Wireless Networks”,IEEE ICC- Ad-hoc and Networking Symposium, 2012.

[11] Linh Nguyen, and Hoc T. Nguyen, "Mobility based network lifetime in wireless networks: A review",12 Aug 2019.

[12] Md. Golam Rashed, M. Hasnat Kabir, Muhammad Sajjadur Rahim, Shaikh Enayet Ullah, "Cluster Based Hierarchical Routing Protocol For Wireless Network",International Journal of Computer and Network Security(IJCNS),Vol. 2, No. 5, May 2010.

[13] Jyoti Singh, Bhanu Pratap Singh, Subhadra Bose Shaw, "A Survey on LEACH-based Hierarchical Routing Protocols in Wireless Network",International Journal of Engineering Research \& Technology (IJERT),Vol. 3 Issue 6, June - 2014.

[14] Gulista Khan, Gaurav Bathla and Wajid Ali, "Minimum Spanning Tree based Routing Strategy for Homogeneous WSN",International Journal on Cloud Computing: Services and Architecture(IJCCSA),Vol.1, No.2, August 2011.

[15] Arun Kumar, Hnin Yu Shwe, Kai Juan Wong, Peter H. J. Chong, "Location-Based Routing Protocols for Wireless Networks: A Survey",Wireless Network, 2017.

[16] Ehsan Ahvar, Mahmood Fathy, "BEAR: A Balanced Energy-Aware Routing Protocol for Wireless Networks",Wireless Network, 2010.

[17] AkoijamPremita, “A Review on Power Efficient Energy-Aware Routing Protocol for Wireless Networks",International Journal of 
Engineering Research \& Technology (IJERT), Vol. 1 Issue 4, June - 2012.

[18] R. Gopinathan, Dr.P. Manimegalai, "Lifespan Enhanced Energy Efficient Cluster Formation and Trusted Multipath Data Transmission for Packet Forwarding in Wireless Network",International Journal of Applied Engineering Research Volume 12, Number 18, 2017.

[19] Shiva Murthy G ,R.J.D'Souza , Varaprasad G, "Reliability Analysis of Route Redundancy Model for Energy Efficient Node Disjoint Multipath Routing in Wireless Networks",International Conference on Modelling, Optimisation and Computing(ICMOC), 2012.

[20] Terence Chung Hsin SIT, Zheng LIU, Marcelo H. ANG Jr., and Winston Khoon Guan SEAH, "Multi-Robot Mobility Enhanced HopCount Based Localization in Ad-Hoc Networks".

[21] Erol Gelenbe, Edith Ngai and Poonam Yadav, "Routing of High-Priority Packets in Wireless Networks".

[22] Xuetao Wei, Nicholas C. Valler, Michalis Faloutsos, Harsha V. Madhyastha, and Ting-Kai Huang, "XLR: Tackling the Inefficiency of Landmark-based Routing in Large Wireless Networks".

\section{Authors Profile}

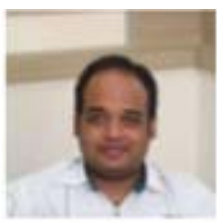

Prof. Sachidanand S. Joshi is an Assistant Professor in the department of Information Science at SDM College of Engineering and Technology, Dharwad, Karnataka, INDIA. He obtained his Bachelor of Engineering from VTU, Belagavi. He received his Master degree in from VTU Belagavi. He is pursuing his Ph.D. from V.T.U., Belagavi-Karnataka, India.

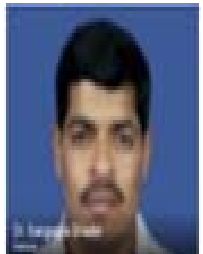

Dr. Sangappa R. Biradar is a Professor in the department of Information Science and Engineering, at S.D.M. College of Engineering and Technology, Dharwad, Karnataka, INDIA. He obtained his Bachelor of Engineering from BLDEA's College of Engineering \& Technology, BIJAPUR. He obtained his Masters of Technology from M.I.T., MAHE-MANIPAL. He received his Ph.D. from JADHAVPUR UNIVERSITY, KOLKATTA, INDIA. He has published many papers in National and International Journals and he got a decade of experience in teaching core papers of Computer Science. His area of interest includes Wireless Sensor Networks and Mobile Ad-Hoc Networks. 